James Foran, Department of Mathematics, University of Missouri-Kansas

City, Kansas City, MO 64110. e-mail: jforan@cctr.umkc.edu

\title{
THE LENGTH OF THE GRAPH OF A ONE TO ONE FUNCTION FROM $[0,1]$ TO $[0,1]$
}

\begin{abstract}
Upper and lower limits for the length of the graph of one to one, onto, Baire one functions from the unit interval to itself are shown to be infinity and one, respectively. Such functions having graphs of large dimension and non-measurable functions are also considered.
\end{abstract}

For the length of a subset $E$ of the plane, we will use the one dimensional Hausdorff measure

$$
\Lambda(E)=\lim _{\delta \rightarrow 0} \inf \sum \operatorname{diam}\left(E_{i}\right)
$$

where the infimum is over all $\left\{E_{i}\right\}_{i=1}^{\infty}$ with $E \subset \cup E_{i}$ and $\operatorname{diam}\left(E_{i}\right)<\delta$. Some well known facts which we will need are:

1. If $E$ is the graph of a continuous function on $[a, b]$ then $\Lambda(E)$ agrees with the length of $E$.

2. If $\operatorname{proj}_{\theta}(E)$ is the perpendicular projection of $E$ onto a line making an angle $\theta$ with the x-axis then $\Lambda\left(\operatorname{proj}_{\theta}(E)\right) \leq \Lambda(E)$.

We will eventually also use the s-dimensional Hausdorff measure of $E$ given by

$$
\Lambda_{s}^{*}(E)=\lim _{\delta \rightarrow 0} \inf \sum(\operatorname{diam}(E))^{s}
$$

where the infimum is as above and $\Lambda_{s}(E)$ replaces $\Lambda_{s}^{*}(E)$ when $E$ is measurable. By the dimension of $E$ will be meant the Hausdorff dimension, $\operatorname{dim}(E)=\inf \left\{s: \Lambda_{s}(E)=0\right\}$. The s-dimensional measure can be estimated by using squares from a sequence of regular nets and we will eventually use nets on the unit square whose squares have vertices with coordinates $m / K^{n}$. See e.g., [1] or [4].

For continuous one to one functions $f$ taking $[0,1]$ onto $[0,1]$ it is well known that $f$ must be monotone and the length of the graph of such an $f$ can be any value in $[\sqrt{2}, 2]$. That the length must be at least $\sqrt{2}$ is due to the fact

Key Words: function, graph, Hausdorff measure

Mathematical Reviews subject classification: 26A21, 28A78

Received by the editors September 6, 1998 
that the graph must have a point on each line perpendicular to $y=x, x \in[0,1]$ (in the event that $f$ is increasing) or $y=1-x, x \in[0,1]$ (if $f$ is decreasing) and thus its length is at least that of its projection on this line segment; that is, is at least $\sqrt{2}$. For the other extreme, any strictly increasing singular function (see e.g. [5] p.101) from $[0,1]$ onto $[0,1]$ will have graph with length 2. Since the derivative of such a function is zero a.e., the set of points on the graph where the derivative is zero has length at least 1 (its projection on the $\mathrm{x}$-axis is almost all of $[0,1])$. But the projection of this set on the y-axis has length 0 (see [5] p.227), and indeed the image of $\left(f^{\prime}(x)=0\right)$ is always of measure 0 . Then the set of points on the graph of $f$ where the derivative is not 0 or does not exist must have length 1 since its projection on the y-axis is of length 1 . But the length of the graph of $f$ is given by $\sup \sum\left(\left|I_{n}\right|^{2}+\left|f\left(I_{n}\right)\right|^{2}\right)^{1 / 2}$ where the supremum is over all partitions $\left\{I_{n}\right\}$ of $[0,1]$. Thus the length is less than or equal to $\sum\left|I_{n}\right|+\left|f\left(I_{n}\right)\right| \leq 2$. And thus the length of the graph of such a function is 2 . That the length can be any value between $\sqrt{2}$ and 2 is easily shown by a function whose graph consists of two line segments connecting $(0,0)$ to $(a, 1-a)$ and $(a, 1-a)$ to $(1,1)$ for appropriately chosen $a \in(0,1)$.

The other extreme of one to one functions from $[0,1]$ onto $[0,1]$ is that of a non-measurable function whose graph has two dimensional Lebesgue outer measure 1. Such a function can be obtained by using the axiom of choice and chosing the points of the graph so there is one point in each $G_{\delta}$ subset of the square whose projection on the $\mathrm{x}$-axis has cardinality $c$. Note that a measurable function must have a graph whose area is 0 (see [5] p.89).

Here we will be interested in relaxing the continuity condition and seeing what is possible for the measure of the graph. A weaker form of continuity, such as Darboux continuity (the intermediate value property), does not result in any new possibilities because a one to one function is Darboux continuous iff it is continuous. If the classes of measurable functions and the Baire classes are considered, it turns out that all the examples needed can be found among the Baire 1 functions. We first consider whether it is possible to obtain a function whose graph has length less than $\sqrt{2}$. Except for the obvious fact that the length of the graph cannot be less than 1, there is no other lower limit on the length of the graph of a one to one function from $[0,1]$ onto $[0,1]$.

Motivation for the construction came from a subset of the plane with interesting properties. Consider the following perfect set contained in an equilateral triangle of side length 1 . Start with the triangle and in each corner place an equilateral triangle whose side length is $1 / 3$. Call these three triangles along with their interiors $E_{1}$. Let $E_{2}$ be the set consisting of nine triangles along with their interiors of side length $1 / 9$ located in the corners of the triangles of $E_{1}$. In general $E_{n}$ consists of $3^{n}$ triangles of side length $3^{-n}$ located in 
the corners of the triangles of $E_{n-1}$. Then $E=\cap E_{n}$ has length less than or equal to 1 because for each natural number $n$ it is contained in $3^{n}$ sets of diameter $3^{-n}$. That the length of $E$ is equal to 1 can be seen by noting that the projection of $E$ onto one of the sides of the original triangle is all of the side and thus the length of $E$ must be at least 1. Actually, what is interesting here is that $E$ projects in each of three directions onto a line segment of length equal to the length of $E$. A natural question is: In how many directions can a set $E$ of finite, non-zero length project onto a set with the same length as $E$ ? (It is easy to observe that the same construction as above using pentagons instead of triangles does not produce a set projecting in any direction onto one of the same length as that of the set.) The fact that a set can project in three directions onto one of the same length as the set suggests the possibility of a function on $[0,1]$ which is one to one and onto $[0,1]$ which has graph of length 1. (For the graph of such a function would be a set which would project onto a set of length one in each of two directions.)

Before constructing a function, we first create a set of length 1 whose projection on each axis is $[0,1]$. The basic idea is to keep the set at each stage closer and closer to a collection of diamonds whose corners are the midpoints of the squares of the previous stage. In particular, a square $Q$ of side length $s$ is divided into $4^{n}$ squares of side length $2^{-n} s$. Then let $A$ be the set consisting of all the points of the subsquares whose interiors meet the boundary of a square whose corners are the midpoints of the sides of $Q$. Then the diameter of the set $A$ is $2 \sqrt{\left(s / 2^{n}\right)^{2}+(s / 2)^{2}}=2 \sqrt{2^{-2 n}+1 / 4} \cdot s$. Note that this number tends to $s$ as $n$ tends to $\infty$. Also note that it is possible to choose $2^{n}$ of these smaller squares which project horizontally and vertically onto the side of the original square. For each $m$ satisfying $0 \leq m \leq 2^{n} / 2$ and each choice of $m$ squares from the $2^{n} / 2$ in the part of $A$ in the upper left quarter of the main square, there is a unique way to choose $2^{n} / 2-m$ from the part of $A$ in the upper right quarter, $m$ from the part of $A$ in the lower right, and $2^{n} / 2-m$ from the part of $A$ in the lower left so that the projections are as required. For a specific example, one can start at the center of the left hand side of the original square and choose $m \leq 2^{n} / 2$ squares going upward and to the right, then starting at the midpoint of the top choose $2^{n} / 2-m$ squares going downward to the right. Starting at the midpoint of the righthand side of the original square one can choose $m$ squares going down to the left and finally $2^{n} / 2-m$ squares starting at the midpoint of the bottom of the original square and proceeding upward and to the left. Specifically, one chooses $2^{n}$ squares whose lower left hand corners in the smaller square correspond to the subsquares of $[0,1] \times[0,1]$ whose lower left hand corners are: $(0,1 / 2),\left(2^{-n}, 1 / 2+2^{-n}\right), \ldots,\left((m-1) 2^{-n}, 1 / 2+(m-1) 2^{-n}\right)$ or $\left(1 / 2,1-2^{-n}\right),\left(1 / 2+2^{-n}, 1-2 \cdot 2^{-n}\right), \ldots,\left(1 / 2+\left(2^{n}-m\right) 2^{-n}, 1-\left(2^{n-1}-m\right) 2^{-n}\right)$ 
or $\left(1-2^{-n}, 1 / 2-2^{-n}\right), \ldots,\left(1-m \cdot 2^{-n}, 1 / 2-m \cdot 2^{-n}\right)$ or $\left(1 / 2-2^{-n}, 0\right), \ldots$, $\left(1 / 2-\left(2^{n-1}-m-1\right) 2^{-n},\left(2^{n-1}-m\right) 2^{-n}\right)$.

Now, let $E_{1}$ consist of $2^{n_{1}}$ squares along with their interiors in $[0,1] \times[0,1]$ each of side length $2^{-n_{1}}$ whose projection on each axis is $[0,1]$ constructed as above. Continuing inductively, let $E_{i}$ consist of $2^{n_{i}}$ squares in each square of $E_{i-1}$ so that the projections horizontally and vertically of these squares are the same as the projection of the square of $E_{i-1}$ containing them. Thus $E_{i}$ projects horizontally and vertically onto $[0,1]$ on the $\mathrm{x}$ and $\mathrm{y}$ axes. Let $E=\cap E_{i}$ and note that each $E_{i}$ is contained in $2^{n_{1}+n_{2}+\ldots+n_{i-1}}$ sets in the squares of $E_{i-1}$ each having diameter $2 \sqrt{2^{-2 n_{i}}+1 / 4} \cdot s$ where $s=2^{-n_{1}-n_{2}-\ldots-n_{i-1}}$. Thus if $\lim \sup n_{i}=\infty$, the length of the set $E=1$.

The set $E$ is a perfect set whose projection onto the $\mathrm{x}$ and $\mathrm{y}$ axis is $[0,1]$ and the length of $E$ is 1 . However, $E$ is not the graph of a function. But $E$ is almost the graph of a function; indeed, if the set of points $E^{\prime}$ of $E$ which have one coordinate in $D$, the set of dyadic rational numbers, is removed from $E$ (there are only countably many such points), the set $E \backslash E^{\prime}$ is the graph of a one to one function from $[0,1]-D$ onto $[0,1]-D$ and it can be defined on the dyadic rationals to be the identity, making it a one to one function from $[0,1]$ onto $[0,1]$ whose graph has length 1 . It is not difficult to see that the image of each open interval differs from a closed set by a countable set and thus is a $G_{\delta \sigma}$ set which implies that the function is in Baire Class 2. (See e.g., [3] p.144.) A slight modification of the construction, keeping the value at the dyadic rationals close to the set $E \backslash E^{\prime}$ results in a function in Baire Class 1. We illustrate this with a specific example.

Example 1. There is a function $g$ in Baire Class 1 whose graph has length 1 (the example will also be constructed so thatg $=g^{-1}$ by constructing the graph of the function symmetric about $y=x$

Construction. One uses the same construction described above but with $m=0$ at each stage. One also redefines the function on the dyadic rationals determined by the edges of the squares at each stage so that they lie inside the squares of the previous stage and map into the set of these same dyadic rationals. Thus, at the first stage $g\left(i / 2^{n}\right)=i / 2^{n}, i=0,1, \ldots, n$. At each successive stage the dyadic rationals corresponding to new edges take on values along the diagonal of the square of the previous stage parallel to $y=x$. In this way the resulting function will satisfy $g=g^{-1}$ and since $g$ will only be discontinuous on the countable set consisting of dyadic rationals, $g$ will be in Baire Class 1.

We now turn to the upper limit for the length. The goal is to produce one to one onto functions with any given length graph among the Baire Class 1 
functions. To do this a method will be described to produce functions whose graphs are of finite length as large as desired. Creating graphs of a given length can then be done, for example, by putting shrunken copies of large graphs in the squares at the various stages of the construction of Example 1. Perhaps it is not surprising that it is also possible to produce such graphs having large dimensions. A construction of a graph of a one to one, onto function with graph of dimension 2 will be sketched. Finally there will be some consideration of non-measurable functions.

While it is worthwhile to draw some pictures of these constructions, the constructions will be given in a strictly numerical fashion.

Example 2. There is a sequence of functions $\left\{g_{K}\right\}$ which are one to one from $[0,1]$ onto $[0,1]$ which are in Baire Class 1 whose graphs have finite length tending to infinity with $K$.

Construction. Given $K \geq 2$ select the sequence of nets on the unit square consisting of squares, half open on the right, whose projections on the axes are of the form $\left\{\left[m / K^{n},(m+1) / K^{n}\right): 0 \leq m<K^{n}\right\}_{n=1}^{\infty}$. Consider $x$ and $y$ in $[0,1)$ written in the K-ary expansion: $x=\sum_{i=1}^{\infty} a_{i} / K^{i}, y=\sum_{i=1}^{\infty} b_{i} / K^{i}$ with $0 \leq a_{i}, b_{i}<K$. (Expressions for numbers which can be written two ways are written terminating in $0^{\prime} s$.) The function $g_{K}$ will be defined by letting $g_{K}(x)=y$ iff for the expansions of $x$ and $y, b_{2}=a_{1}, a_{2 n+1}=b_{2 n-1}, n>0$ and $b_{2 n}=a_{2 n-2}(n>1)$. The resulting function is one to one and belongs to Baire Class 1 because the set of discontinuities is at most countable (the points $x$ where the $a_{i}$ defining $x$ terminate in $0^{\prime} s$ ). The graph of $g_{K}$ can be contained in $K^{n+1}$ squares of side length $K^{-n}$ where $n$ is odd and $n>2$; namely, those squares with lower left hand corner given by $\left(\sum_{i=1}^{n} a_{i} / K^{i}, \sum_{i=1}^{n} b_{i} / K^{i}\right)$ with $b_{1}, \ldots, b_{n-1}$ determined by $a_{1}, \ldots, a_{n-2}$ and $a_{n}$ and both $a_{n-1}$ and $b_{n}$ taking any value from 0 to $K-1$. Thus the Hausdorff measure of the graph is at most $K \sqrt{2}$. That the length of the graph is at least $K / \sqrt{2}$ can be seen by considering the $K$ parts of the graph above each of the intervals $[m / K,(m+1) / K)$. First note that each of these sets can be obtained by a translation of the points above $[0,1 / K)$ since $f\left(. a_{1} a_{2} a_{3} \ldots a_{n}\right)=f\left(.0 a_{2} a_{3} \ldots a_{n}\right)+a_{1} K^{-2}$. To complete the argument, we show that every $z \in[0,1)$ is of the form $x+f(x)$ for suitable $x \in[0,1 / K)$ and thus the graph above $[0,1 / K)$ projects perpendicularly on the line $y=x$ onto an interval of length at least $1 / \sqrt{2}$. To see that each $z \in[0,1)$ is of the form $x+f(x)$ for some $x \in[0,1 / K)$, note that if $x=.0 a_{2} a_{3} a_{4} a_{5} a_{6} a_{7} a_{8} \ldots$, then we have $f(x)=._{3} 0 a_{5} a_{2} a_{7} a_{4} a_{9} a_{6} \ldots$ and it follows that $x+f(x)=(1+$ $\left.K^{2}\right)\left(a_{3} K^{-3}+a_{2} K^{-4}+a_{5} K^{-5}+a_{4} K^{-6}+a_{7} K^{-7}+a_{6} K^{-8}+\ldots\right.$. Thus if $z \in[0,1)$ and $y=z /\left(1+K^{2}\right)$, and if $y=\sum b_{n} K^{-n}$ where, necessarily, $b_{1}=b_{2}=0$, and if $a_{n}=b_{n+2}$ when $n$ is even and $a_{n}=b_{n}$ when $n$ is odd, then $z=x+f(x)$ where $x=\sum a_{n} K^{n} \in[0,1 / K)$. 
Regarding the size of the graph, the example below shows how it is possible to create one to one, onto, Baire one functions whose graph has large dimension. We sketch how to produce a graph whose dimension should be 2 . We show only that its box dimension is 2. (Cf. [2] p.38) That is, it will be shown that for every fixed $s<2$, the limit of the sums of the diameters to the power $s$ of the binary net elements which meet the graph is infinite.

The natural coverings of the graph we will produce are as follows:

At each stage, the interval $[0,1]$ is to be divided into $2^{N_{k}}$ intervals. At the first stage the graph will be contained in the unit square considered as $2^{4}$ squares of side length $2^{-2}$ yielding a total area of 1 . At the second stage there will be $2^{30}$ squares distributed equally among the squares of the previous stage of side length $2^{-16}$ for a total area of $2^{-2}$ (here the total area at this stage is the side length at the previous stage). At the $\mathrm{N}^{t h}$ stage, there will be $2^{M_{N}}$ squares of side length $2^{-K_{N}}$ for a total area of $2^{-K_{N-1}}$ where $M_{N}=$ $2^{(N+1)^{2}} \cdot 2-2^{N^{2}}=2^{N^{2}}\left(2^{2 n+2}-1\right)$ and $K_{N}=2^{(N+1)^{2}}$. If these squares are considered as approximating the dimension of the graph, then for fixed $s$ and large $N$, we have $2^{M_{N}}\left(2^{-K_{N}}\right)^{s} \leq 1$ iff $M_{N} \leq s \cdot K_{N}$ iff $s \geq 2-1 / 2^{2 N+1}$ which implies that this holds for all large $N$ iff $s=2$. (Actually, the referee has pointed out that for $K_{N}$ any strictly increasing sequence of positive integers for which $K_{N}>2 N$ holds for each $N$ and $K_{N}$ has a strictly higher rate of growth than $N$ would work.)

The graph of a one to one onto function can be made requiring the number of squares of these magnitudes by considering $x=\sum a_{i} / 2^{i}$ and $y=\sum b_{i} / 2^{i}$ with numbers having two representations written with terminating $0^{\prime} s$. Consider the numbers of the form $K_{N}$ and let $\left\{B_{N}\right\}$ be all other numbers written in their natural order. Let $f(x)=y$ if $b_{K_{N}}=a_{B_{N}}$ or if $b_{B_{N}}=a_{K_{N}}$ in the binary expansions for $x$ and $y$. Then the number of squares at the $M_{N} t h$ stage needed to cover the graph of $f$ will be of the order given above. To see this, consider the squares from the net whose side lengths are $2^{-n}$ which meet the graph of the function. These are the squares whose lower left hand corner $(x, y)$ is of the form $x=. a_{1} a_{2} \ldots a_{n}$ where there are $n$ choices of 0 's or 1 's to determine $\mathrm{x}$ and thus at least $n-K_{i-1}$ choices of 0 's and 1's for $y$ where $n$ is the least integer larger than $K_{i-1}$. That is, when $n=K_{N}$, there are at least $M_{N}=2 K_{N}-K_{N-1}$ choices for squares of side length $2^{-K_{N}}$ which meet the graph. Considering other covers from the sequence of binary nets, at each stage after the $K_{N}$ th and before the $K_{N+1} s t$ the number of squares needed from a given net is 4 times the number needed at the previous stage. Each time 4 times the number of squares is required, the estimate of the $s$ dimension increases. This continues to the $M_{N+1} s t$ stage. Since the estimate of the dimension using the net elements at the $M_{N} t h$ stage is less then that of 
the $M_{N+1}$ st stage, for each $s<2$, and since this estimate approaches infinity for each fixed $s$ less than 2 , it follows that the graph has box dimension 2 .

It remains to determine how small the graph of a one to one function $f$ from $[0,1]$ onto $[0,1]$ can be if $f$ is non-measurable. Since the graph could simply be non-measurable on a small subinterval and then have a graph of length as close to one as desired, we will be interested only in such $f$ which are not measurable on any perfect subset of $[0,1]$ of positive measure. The next theorem shows that it is not possible to have such a non-measurable function with graph of length 1 . The example which follows it shows how to produce a one to one function, not measurable on any perfect subset of positive measure whose graph has outer one dimensional measure 2 .

Theorem. If $f$ is one to one from $[0,1]$ onto $[0,1]$ and the length of the graph of $f$ is 1 , then $f$ is a measurable function. Also, for each interval $I \subset[0,1]$, $m(f(I))=|I|$.

Proof. Let $g=f^{-1}$ and $J_{0}=[c, d] \subset[0,1]$. Let $J_{1}=[0, c]$ and $J_{2}=[d, 1]$ and let $E_{i}$ be the graph of $g$ on the intervals $J_{i}$ with $i=0,1,2$. Then $\Lambda^{*}\left(E_{i}\right) \geq\left|J_{i}\right|$ and

$$
\Lambda^{*}\left(E_{1}\right)+\Lambda^{*}\left(E_{0}\right)+\Lambda^{*}\left(E_{2}\right)=1
$$

and outer length measure adds on these three sets. It is not possible that $\Lambda^{*}\left(E_{0}\right)>\left|J_{0}\right|$ for if it were, $\left|J_{1}\right|+\left|J_{0}\right|+\left|J_{2}\right|$ would be less than 1 , a contradiction. But then $m^{*}\left(f^{-1}\left(J_{i}\right)\right) \leq \Lambda^{*}\left(E_{i}\right)=\left|J_{i}\right|$ for $i=0,1,2$. Therefore, $\sum m^{*}\left(f^{-1}\left(J_{i}\right)\right) \leq 1$. But since the opposite inequality always holds, $\sum m^{*}\left(f^{-1}\left(J_{i}\right)\right)=1$ and $f^{-1}\left(J_{0}\right)$ is measurable and since $J_{0}$ is an arbitrary interval in the range of $f$, it follows that $f$ is a measurable function. Furthermore, $g$ is measurable and applying the above to $g$ we have for each $I \subset[0,1]$ that $m(f(I))=|I|$.

Example 3. There is a function which is one to one from $[0,1]$ onto $[0,1]$ which is non-measurable on each perfect subset of positive measure and has graph of outer linear measure equal to 2.

Construction. Let $g$ be the one to one function from $[0,1]$ onto $[0,1]$ described above with $g$ in Baire Class $1, g(1 / 2)=1 / 2$, and $g=g^{-1}$. Note that $1-g(x)=g(1-x)$ at each $x \in[0,1]$. Choose $S$ so that both $S$ and $S^{c}$ are totally imperfect subsets of $[0,1]$ satisfying $x \in S$ iff $g(x) \in S, 1-x \in S$ and $g(1-x) \in S$. This can be done by well ordering the non empty perfect sets contained in $[0,1]$ and choosing elements $x$ for $S$ and for $S^{c}$ at each stage with $g(x), 1-x, g(1-x)$ also in $\dot{S}$ or $S^{c}$, respectively. Then let $f(x)=g(x)$ if $x \in S$ and $f(x)=1-g(x)$ if $x \in S^{c}$. Then $f^{-1}([0,1 / 2])$ is the union of $S \cap[0,1 / 2]$ and $S^{c} \cap[1 / 2,1]$ and thus it has non-measurable intersection with each perfect 
set of positive measure. To see that $f$ is one to one, suppose $f(a)=f(b)$ with $a \neq b$. Note that $g(x)=1-g(x)$ iff $g(x)=1 / 2$, and then $x=1 / 2$. Since $g$ is one to one, if $f(a)=g(a)$, then $f(b)=1-g(b)$ or if $f(a)=1-g(a)$, then $f(b)=g(b)$. In the former case $a \in S^{c}$ and $b \in S$; in the latter $a \in S$ and $b \in S^{c}$; in neither case can $f(a)$ equal $f(b)$ since $a \in S$ iff $g(a) \in S, 1-a \in S$ and $1-g(a)=g(1-a) \in S$.

The author wishes to thank the referee for his patience with the two drafts of this paper and for his many helpful suggestions and corrections.

\section{References}

[1] Falconer, K.J., The Geometry of Fractal Sets, Cambridge University Press, 1985.

[2] Falconer, K.J., Fractal Geometry, Wiley, 1990.

[3] Goffman, C., Real Functions, Prindle, Weber, Schmidt, 1953.

[4] Rogers, C.A., Hausdorff Measures, Cambridge University Press, 1970.

[5] Saks, S., Theory of the Integral, Dover Publications, 1964. 